\title{
Multiparametric cystoscopy: is the future here yet?
}

\author{
Hamed Ahmadi, Siamak Daneshmand \\ Department of Urology, University of Southern California, Norris Comprehensive Cancer Center, Los Angeles, CA, USA \\ Correspondence to: Siamak Daneshmand, MD. Associate Professor of Urology (Clinical Scholar), USC Department of Urology, Norris Comprehensive \\ Cancer Center, 1441 Eastlake Avenue, Suite 7416, Los Angeles, CA 90089-2211, USA. Email: daneshma@med.usc.edu. \\ Comment on: Kriegmair MC, Rother J, Grychtol B, et al. Multiparametric Cystoscopy for Detection of Bladder Cancer Using Real-time Multispectral \\ Imaging. Eur Urol 2020;77:251-9.
}

Submitted Jun 18, 2020. Accepted for publication Jun 29, 2020.

doi: $10.21037 /$ tau-20-1012

View this article at: http://dx.doi.org/10.21037/tau-20-1012

\section{Introduction}

Since the invention of first working cystoscope in 1878 in Germany, its technology has gone through major revolutions and white light cystoscopy (WLC) is now considered the gold standard endoscopic procedure for diagnosis and local staging of bladder cancer (1). For a long time, WLC was the only option available for endoscopic staging, treatment and surveillance of bladder tumor. However, it has significant shortcomings such as poor sensitivity for detecting small papillary tumors or carcinoma in situ (CIS). The sensitivity of WLC in detecting any bladder tumor, regardless of characteristic or grade, is only $76 \%$ and its detection rate falls down to disappointing range of 55-68\% for CIS lesions (2). Urine cytology still remains a routine part of diagnostic and surveillance cystoscopy. However, its overall sensitivity for detecting bladder tumor ranges between $59 \%$ and $62 \%$ and it missed almost $20 \%$ of all high-grade tumors (3).

Introduction of enhanced endoscopic imaging technologies opened a new horizon in management of non-muscle invasive bladder cancer (NMIBC). These are generally categorized into macroscopic, microscopic and molecular technologies based on their scope (4). Macroscopic technologies, such as photodynamic diagnosis, also referred to as blue light cystoscopy (BLC), and narrow band imaging (NBI) have already been tested in several multi-institutional randomized trials or prospective studies and are currently recommended as adjunct procedure to WLC to improve detection rate of bladder cancer and improve quality of resection in major Urologic guidelines $(5,6)$. Images produced by technologies such as BLC or NBI could not be viewed parallel with $\mathrm{WL}$ images on the same monitor and therefore, surgeons generally switch between enhanced and WL images during transurethral resection of bladder tumor (TURBT) to identify additional lesions visible only on enhanced images and also to assure complete resection of tumor and its margins. The ability to visualize enhanced images either individually and or overlaid with $W \mathrm{~L}$ images at the same time is an intriguing idea.

\section{Real time multi spectral imaging}

Kriegmair et al., in a proof-of-concept study, introduced an enhanced imaging system that allows simultaneous visualization of enhanced images parallel or overlaid with WL images during TURBT using computer assisted image processing technology (7). This system uses 6 different imaging modalities including WL, BLC, NBI, auto fluorescence $(\mathrm{AF})$, protoporphyrin IX-fluorescence (PpIX-F), and multiparametric (MP). rMSI system consists of a metal-oxide-semiconductor camera with a C-mount, a video adapter with a glued optical filter, and a light source. The camera and light source are controlled via a microcontroller which is interfaced with a software on a computer that does basic image processing and streams the video real time on a monitor. Initially, the live image that surgeon could see on the monitor during the procedure was MP images obtained via digital fusion of WLC, NBI, BLC. PpIX-F and AF images were derived from the BLC using more complex image processing post intervention. However, they were able to add those images to live MP image as well during the course of the study. Images from all different modalities as well as MP images were then reviewed by two urologist after the procedure and 
scored based on level of suspicion for malignancy from 0 (not suspicious at all) to 4 (very suspicious). A total of 10 patients were included in the study and a total of 31 lesions were resected using this system. They did not report any technical issues or complications related to the new imaging system. They also found that rMSI did not miss any lesions detected already by PDD and MP image was the only image modality which was scored 2 or 3 for all the images. They also reported zero probability of failing to identify a suspicious lesion on MP images.

Clearly, these findings are very interesting. As expected in a proof of concept study, a highly selected group of patients, mostly low-grade papillary lesions, were enrolled but it will be interesting to see if combination of different macroscopic technologies could improve specificity in post BCG setting in the next phases of the study. With regards to image interpretation, it is important to mention that two urologist who interpreted and scored the MP images in this study were provided with post-procedure, more enhanced version of images rather than real-time images shown on the monitor during the procedure. This obviously questions the validity of the scoring system they used in this study.

It is also important to understand how this fairly complex fusion and image processing technology compares with currently available systems. This editorial provides a opportunity to review some of the latest enhanced optical imaging technologies. As mentioned earlier, enhanced optical imaging technologies are generally classified into three board categories: macroscopic, microscopic and molecular imaging.

\section{Macroscopic enhanced imaging technologies}

Real time multispectral image technology essentially combines 3 different enhanced macroscopic modalities.

\section{$B L C$}

In this technology, hexaminolevulinate (HAL; known as Cysview in the US and Hexvix in Europe) is used which is a heme precursor and preferentially accumulates protoporphyrin IX and other photoactive porphyrins in the mitochondria of neoplastic tissue and emit fluorescence when exposed to with blue light in the wavelength between 375 and $440 \mathrm{~nm}$. The role of BLC in improving management of NMIBC is well supported by high level evidence $(2,8)$. Data from randomized clinical trial and meta-analysis suggest detection of additional $29 \%$ papillary tumors and $24 \%$ reduction in recurrence rate at 12 months by BLC compared to WLC $(8,9)$. Another important role of BLC is marked increase in detection rate of CIS up to $43 \%$ based on the results of multicenter prospective study (2). This technology is also available for flexible office blue light cystoscopy (FBLC) as well, and the results of phase III prospective trial, conducted in 17 centers in US, demonstrated similar improvements in cancer detection. Out of 103 patients who were taken to operating room due to suspicious lesion found on flexible cystoscopy, $21 \%$ had a lesion only visible by FBLC. Also, they reported 5 patients with CIS lesions that were only detectable by FBLC. Interestingly, all those patients had negative cytology (10).

\section{$N B I$}

This technology uses a light source that filters white light into 2 wavelengths $(415$ and $540 \mathrm{~nm}$ ) that are strongly absorbed by hemoglobin, and therefore helps differentiating vascularized lesions from normal and benign urothelium. One of the advantages of NBI is that it does not require preoperative instillation contrast. However, interpretation of images is inherently subjective and relies on individual ability to detect visual change in the vasculature around the suspicious areas. Therefore, bleeding during TURBT could significantly reduce enhanced visualization and subsequently, NBI's accuracy in detecting additional lesions or assessing adequate resection $(11,12)$. Additionally, there is lack of consistent evidence suggesting an improved tumor recurrence or progression by use of NBI. Early results from multicenter randomized trial of NBI-assisted TURBT versus WLC TURBT (CROES trial) did not show any significant difference in overall recurrence free survival (13). False positive rate is as high as $36 \%$ and the ability to differentiate post BCG reaction from recurrence remains a question (4).

\section{Auto-florescence (AF)}

Similar to BLC, this technology is based on fluorescent light tissue emission phenomenon. The difference is that AF mainly targets endogenous fluorophores such as tryptophan and collagen to differentiate malignant and healthy tissues. Therefore, there is no need for preoperative instillation of HAL. The ideal wavelength for this technology seems to be within ultraviolet (UV) spectrum 
and therefore, initial studies of AF used UV-light source for cell excitation. However, it raises safety concerns and hampers its widespread use (11). Using a longer wavelength within the visible spectrum, Kriegmair et al. showed $\sim 10 \%$ improvement in detection rate of malignant lesions using AF compared to WLC. However, specificity of AF was poor with false positive rate close to $46 \%$ (14).

\section{rMSI versus individual enbanced macroscopic imaging}

Although Kriegmair et al. made the need for switching between WL and enhanced images during TURBT their main argument for developing live imaging fusion technology, from personal experience, this does not seem to be a major obstacle. The biggest potential advantage of rMSI system, however, could be minimizing false positive rate and inter observer variability in image interpretation. Some of the argued limitations of macroscopic enhanced imaging technologies especially with BLC such as the need for preoperative catheterization and cost associated with purchase of the endoscopic equipment are definitely not addressed by rMSI. It is difficult to imagine that this complex system would be less costly than BLC.

\section{Storz professional image enhancement system}

Also known as IMAGE1 S and SPIES, this is probably the closest available technology to rMSI as a multimodal imaging technology using different image processing algorithms. IMAGE1 S offers several image enhancement modalities including spectra A, spectra B, chroma and clara mode (15). Spectra A and B use color tone shift algorithms to increase the contrast. Chroma enhances the sharpness of the image while Clara provides greater visibility of darker regions within the image. This system provides a standard WL image in parallel to enhanced images simultaneously and each image modality is specifically designed and best implicated for different clinical scenarios (16). Results of a pilot study suggest that Clara and Chroma or Spectra B combination during cystoscopy should be used given the less variability in image interpretation (17).

IMAGE $1 \mathrm{~S}$ does obviate the need for preoperative instillation of any exogenous florescent material but contrary to rMSI, it does not provide real time MP images overlaid with WL images. There is limited data to show the efficacy of this system and we are still awaiting the results of large prospective international trial by CROES (18). Therefore, it is early to make any comparison between these two modalities in terms of outcome.

\section{Microscopic imaging modalities}

As opposed to macroscopic modalities which have similar overall field of view and spatial resolutions compared to WLC, microscopic modalities provide cellular resolutions as well as subsurface imaging which could theoretically provide histologic cancer characterization including grading and staging. On the flip side, there could a steep learning curve for real time interpretation of high-resolution images (19).

\section{Optical coberence tomography (OCT)}

This technique provides real-time, cross-sectional images with up to $2 \mathrm{~mm}$ depth of penetration and 10-20 $\mu \mathrm{m}$ spatial resolution which is 10-25 times greater than highfrequency ultrasound (20). Under OCT, normal bladder wall anatomy from urothelium to muscularis propria is nicely delineated in a layer by layer fashion which will be distorted in the presence of malignant lesions with or without invasion. This modality has shown very high sensitivity for detection of any bladder tumor including invasive lesions and CIS (20). Unfortunately, this technology is unable to provide any grading information and its real-world practice might be limited partly due to the need for scanning of the entire bladder and technical consideration (19).

\section{Confocal laser endomicroscopy (CLE)}

CLE provides the highest resolution $(1-5 \mu \mathrm{m})$ of all the modalities discussed so far, comparable to conventional histopathologic examination, which allows grading of the malignant lesion (21). The diameter is the probe used for this technology varies between 0.85 to $2.6 \mathrm{~mm}$, allowing passage through standard rigid cystoscopes. Contrary to OCT, CLE would require preoperative contrast administration. Fluorescein, a FDA-approved contrast agent with high safety profile, is currently administered before CLE both intravesically or intravenously. The probe used with OCT needs to be in direct contact with the tissue which could be challenging for some locations such as anterior bladder wall or dome using resectoscope. However, smaller size probe that could be passed through flexible cystoscope might be used in these circumstances. Also, its small field of view makes it impractical to use CLE to scan the entire bladder (11). 


\section{Raman spectroscopy (RS)}

This technology uses tissue specific spectra which are generated when intrinsic chemical bonds are excited with light, and an optical contrast is generated depending on the tissue properties. There is no need for exogenous contrast instillation prior to cystoscopy. Early studies suggested sensitivity of $85-86 \%$ and specificity of $79-100 \%$ for differentiating bladder tumor from normal urothelium. Recent meta-analysis suggested a pooled sensitivity and specificity of $92 \%$ and $94 \%$, respectively (22). Time shift up to 5 seconds, narrow field of view, and weak signals are major limitations of this technique (4).

\section{Combination with other enbanced imaging techniques}

Each one of these microscopic modalities have been studied in combination with either macroscopic or molecular imaging techniques. Recent meta-analysis showed that combination of OCT with florescence imaging technologies such as BLC could further improve its diagnostic accuracy and avoid unnecessary biopsies (23). The results of ex vivo study of combining OCT and BLC, however, had been disappointing as HAL cannot provide a clear cellular architecture due to due to cytoplasmic localization (24). It has been suggested that combination of RS and molecular targeting nanoparticles, referred to as surface-enhanced RS, can significantly improve sensitivity and specificity for detection of bladder tumors through signal amplification and molecular mapping (4).

\section{Molecular imaging}

Molecular imaging technology by definition is a multimodal imaging modality. In this technology, fluorescently labeled binding agents such as antibodies, peptides, or small molecules are being captured using macroscopic enhanced imaging devices. Such agents ideally should bind to highly expressed, favorably specific targets for bladder tumor, have good safety profile and fitting pharmacokinetics including high stability in urine. The most extensively studied agent is CD47 antibody (anti-CD47) which targets a surface marker (CD47), expressed on more than $80 \%$ of bladder tumor cells. Small ex vivo study suggest that the combination of CD47-targeted imaging and BLC could increase the specificity for bladder tumor detection to $90.5 \%$ (25). A class of membrane-binding peptides, known as $\mathrm{PH}$ low insertion peptides (pHLIPs), has also been studied as potential targeting agent. Ex vivo study on cystectomy specimens showed that when pHLIPs conjugated to indocyanine green applied to specimens and scanned using clinical grade near-infrared fluorescence imaging system (Firefly ${ }^{\mathrm{TM}}$ ), it was able to identify bladder tumors with $100 \%$ sensitivity regardless of grading or any other histologic subtypes. However, false positive rate was as high as $20 \%$ (19). Evidently, widespread clinical usage of these modalities in near future seems implausible pending robust clinical data showing their efficacy and safety.

\section{Conclusions}

Although rMSI provides an exciting opportunity for surgeons to view a real time fusion enhanced image on the monitor at the time of resection, BLC and NBI still present the most viable options for enhanced optical imaging supported by highest level of evidence. As Kriegmair et al. mentioned in the discussion, rMSI could potentially be combined with molecular imaging technologies. Ideally, a MP cystoscopy should combine all 3 different categories of enhanced imaging to improve the detection rate, provide histologic characteristics such as staging and grading, and allow molecular details about biological processes that may precede any microscopic or macroscopic changes. Technical issues, associated cost, steep learning curve and most importantly clinical efficacy in real-world practice present major obstacles in the way of widespread clinical usage of these novel techniques in near future.

\section{Acknowledgments}

Funding: None.

\section{Footnote}

Provenance and Peer review: This article was commissioned by the editorial office, Translational Andrology and Urology. The article did not undergo external peer review.

Conflicts of Interest: Both authors have completed the ICMJE uniform disclosure form (available at http://dx.doi. org/10.21037/tau-20-1012). SD reports personal fees from Photocure, outside the submitted work. HA has no conflicts of interest to declare.

Ethical Statement: The authors are accountable for all 
aspects of the work in ensuring that questions related to the accuracy or integrity of any part of the work are appropriately investigated and resolved.

Open Access Statement: This is an Open Access article distributed in accordance with the Creative Commons Attribution-NonCommercial-NoDerivs 4.0 International License (CC BY-NC-ND 4.0), which permits the noncommercial replication and distribution of the article with the strict proviso that no changes or edits are made and the original work is properly cited (including links to both the formal publication through the relevant DOI and the license). See: https://creativecommons.org/licenses/by-nc-nd/4.0/.

\section{References}

1. Reuter MA, Reuter HJ. The development of the cystoscope. J Urol 1998;159:638-40.

2. Daneshmand S, Bazargani ST, Bivalacqua TJ, et al. Blue light cystoscopy for the diagnosis of bladder cancer: Results from the US prospective multicenter registry. Urol Oncol 2018;36:361.e1-6.

3. Breen V, Kasabov N, Kamat AM, et al. A holistic comparative analysis of diagnostic tests for urothelial carcinoma: a study of Cxbladder Detect, UroVysion(R) FISH, NMP22(R) and cytology based on imputation of multiple datasets. BMC Med Res Methodol 2015;15:45.

4. Schubert T, Rausch S, Fahmy O, et al. Optical improvements in the diagnosis of bladder cancer: implications for clinical practice. Ther Adv Urol 2017;9:251-60.

5. Babjuk M, Burger M, Comperat EM, et al. European Association of Urology Guidelines on Non-muscleinvasive Bladder Cancer (TaT1 and Carcinoma In Situ) 2019 Update. Eur Urol 2019;76:639-57.

6. Chang SS, Bochner BH, Chou R, et al. Treatment of Nonmetastatic Muscle-Invasive Bladder Cancer: American Urological Association/American Society of Clinical Oncology/American Society for Radiation Oncology/ Society of Urologic Oncology Clinical Practice Guideline Summary. J Oncol Pract 2017;13:621-5.

7. Kriegmair MC, Rother J, Grychtol B, et al. Multiparametric Cystoscopy for Detection of Bladder Cancer Using Real-time Multispectral Imaging. Eur Urol 2020;77:251-9.

8. Grossman HB, Gomella L, Fradet Y, et al. A phase III, multicenter comparison of hexaminolevulinate fluorescence cystoscopy and white light cystoscopy for the detection of superficial papillary lesions in patients with bladder cancer. J Urol 2007;178:62-7.

9. Burger M, Grossman HB, Droller M, et al. Photodynamic diagnosis of non-muscle-invasive bladder cancer with hexaminolevulinate cystoscopy: a meta-analysis of detection and recurrence based on raw data. Eur Urol 2013;64:846-54.

10. Daneshmand S, Patel S, Lotan Y, et al. Efficacy and Safety of Blue Light Flexible Cystoscopy with Hexaminolevulinate in the Surveillance of Bladder Cancer: A Phase III, Comparative, Multicenter Study. J Urol 2018;199:1158-65.

11. Liu JJ, Droller MJ, Liao JC. New optical imaging technologies for bladder cancer: considerations and perspectives. J Urol 2012;188:361-8.

12. Mukherjee P, George AJP, Yadav BK, et al. The Impact of Narrow Band Imaging in the Detection and Resection of Bladder Tumor in Transitional Cell Carcinoma of the Bladder: A Prospective, Blinded, Sequential Intervention Randomized Controlled Trial. Urology 2019;128:55-61.

13. Naito S, Algaba F, Babjuk M, et al. The Clinical Research Office of the Endourological Society (CROES) Multicentre Randomised Trial of Narrow Band ImagingAssisted Transurethral Resection of Bladder Tumour (TURBT) Versus Conventional White Light ImagingAssisted TURBT in Primary Non-Muscle-invasive Bladder Cancer Patients: Trial Protocol and 1-year Results. Eur Urol 2016;70:506-15.

14. Kriegmair MC, Honeck P, Theuring M, et al. Widefield autofluorescence-guided TUR-B for the detection of bladder cancer: a pilot study. World J Urol 2018;36:745-51.

15. Emiliani E, Talso M, Baghdadi M, et al. Evaluation of the Spies (TM) modalities image quality. Int Braz J Urol 2017;43:476-80.

16. Mari A, Abufaraj M, Gust KM, et al. Novel endoscopic visualization techniques for bladder cancer detection: a review of the contemporary literature. Curr Opin Urol 2018;28:214-8.

17. Kamphuis GM, de Bruin DM, Brandt MJ, et al. Comparing Image Perception of Bladder Tumors in Four Different Storz Professional Image Enhancement System Modalities Using the iSPIES App. J Endourol 2016;30:602-8.

18. Gravas S, Stenzl A. The Storz professional image enhancement system(spies) nonmuscle-invasive bladder cancer study:a multicenter international randomized controlled study. J Endourol 2014;28:1254-5.

19. Kiss B, Marcq G, Liao JC. Optical and Cross-Sectional 
Imaging Technologies for Bladder Cancer. Cancer Treat Res 2018;175:139-63.

20. Pearce S, Daneshmand S. Enhanced Endoscopy in Bladder Cancer. Curr Urol Rep 2018;19:84.

21. Lucas M, Liem E, Savci-Heijink CD, et al. Toward Automated In Vivo Bladder Tumor Stratification Using Confocal Laser Endomicroscopy. J Endourol 2019;33:930-7.

22. Jin H, Lin T, Han P, et al. Efficacy of Raman spectroscopy in the diagnosis of bladder cancer: A systematic review and meta-analysis. Medicine (Baltimore) 2019;98:e18066.

Cite this article as: Ahmadi H, Daneshmand S. Multiparametric cystoscopy: is the future here yet? Transl Androl Urol 2021;10(1):1-6. doi: 10.21037/tau-20-1012
23. Schmidbauer J, Remzi M, Klatte T, et al. Fluorescence cystoscopy with high-resolution optical coherence tomography imaging as an adjunct reduces false-positive findings in the diagnosis of urothelial carcinoma of the bladder. Eur Urol 2009;56:914-9.

24. Marien A, Rock A, Maadarani KE, et al. Urothelial Tumors and Dual-Band Imaging: A New Concept in Confocal Laser Endomicroscopy. J Endourol 2017;31:538-44.

25. Pan Y, Volkmer JP, Mach KE, et al. Endoscopic molecular imaging of human bladder cancer using a CD47 antibody. Sci Transl Med 2014;6:260ra148. 